\title{
Impact of Metabolic Surgery on Renal Injury in Pre-Clinical Models of Diabetic Kidney Disease
}

\author{
William P. Martin ${ }^{a}$ Carel W. le Roux ${ }^{a, b}$ Neil G. Docherty ${ }^{a}$

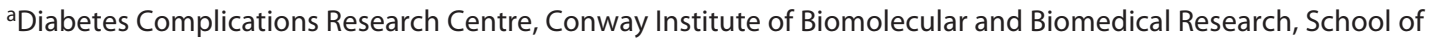 \\ Medicine, University College Dublin, Dublin, Ireland; bivision of Investigative Science, Imperial College London, \\ London, UK
}

\section{Keywords}

Obesity - Type 2 diabetes - Diabetic kidney disease $\cdot$ Chronic kidney disease - Albuminuria - Metabolic surgery · Zucker diabetic fatty rats

\begin{abstract}
Background: Surgical approaches to the treatment of obesity and type 2 diabetes, most notably the Roux-en-Y gastric bypass (RYGB) procedure, have been shown to be renoprotective, reducing the incidence of albuminuria and endstage kidney disease over 15- to 20-year follow-up in patients with obesity. The tissue level effects of metabolic surgery on the diabetic kidney are not easily interrogated in clinical samples. However, elucidation of the cellular and molecular basis for the renoprotective effects of metabolic surgery is now emerging from a body of pre-clinical work in rodent models of diabetic kidney disease (DKD). Summary: Experimental metabolic surgery (RYGB, sleeve gastrectomy [SG], Roux-en-Y oesophagojejunostomy, and duodenojejunal bypass) exerts a pronounced albuminuria-lowering effect in rat models of DKD. Following RYGB in the Zucker diabetic fatty rat, glomerular histology is improved as demonstrated by reductions in podocyte stress, glomerulomegaly, and glomerulosclerosis. Glomerular ultrastructure improves after RYGB and after SG, manifested by quantifiable reduc-
\end{abstract}

tions in podocyte foot process effacement. The transcriptional programme underpinning these structural improvements has been characterized at the pathway level using RNA sequencing and is associated with a significant reduction in the activation of inflammatory and fibrotic responses. Key Messages: Experimental metabolic surgery reduces biochemical, histological, and molecular indices of DKD. These pre-clinical data support a growing interest in the potential utility of metabolic surgery as a therapeutic approach to slow renal functional decline in patients with obesity and DKD.

(c) 2020 S. Karger AG, Basel

\section{Introduction}

Diabetic kidney disease (DKD) is the leading cause of end-stage kidney disease (ESKD) [1]. Although there have been recent advances in medical therapy for DKD, most notably sodium-glucose co-transporter- 2 inhibitors and glucagon-like peptide- 1 receptor agonists, it remains a progressive disease despite intensive outpatient management by nephrologists and diabetologists [2-4]. Most

Contribution from the CME course of the DIABESITY Working Group of the ERA-EDTA, Alcorcón (Madrid), Spain, November 15-16, 2019.

$\begin{aligned} & \text { karger@karger.com } \\ & \text { www.karger.com/nef }\end{aligned}$
Karger ${ }^{\prime /}$

William P. Martin

Diabetes Complications Research Centre Conway Institute of Biomolecular and Biomedical Research, School of Medicine, University College Dublin Belfield, Dublin D04 V1W8 (Ireland)

william.martin@ucd.ie 
of the excess mortality attributable to diabetes occurs in people with kidney disease [5]. Cardiovascular mortality rates increase proportionally with DKD stage and, in particular, are unacceptably high in people with ESKD [6]. Therefore, prevention of progression of DKD to ESKD is of critical importance.

Obesity is common amongst people with chronic kidney disease (CKD); for example, the prevalence of obesity was $44.1 \%$ among adults with CKD in the USA during 2011-2014 and 35.3\% in an Irish tertiary nephrology centre in 2018-2019 [7, 49]. Metabolic surgery is an effective means of inducing sustained weight loss and plays a central role in the management of patients with severe obesity (body mass index $[\mathrm{BMI}] \geq 40 \mathrm{~kg} / \mathrm{m}^{2}$ ) with or without type 2 diabetes. However, given that less severe obesity $\left(\mathrm{BMI}<40 \mathrm{~kg} / \mathrm{m}^{2}\right)$ is more prevalent among patients with DKD and other microvascular complications of type 2 diabetes [49], and much of the beneficial end-organ impact of metabolic surgery occurs independently of weight loss, there is a growing interest in the role of metabolic surgery in patients with type 2 diabetes and less severe obesity [8]. Indeed, the Microvascular Outcomes after Metabolic Surgery randomized controlled trial, which demonstrated that Roux-en-Y gastric bypass (RYGB) surgery is a more effective means of achieving remission of albuminuria at 24 months than best medical treatment, selectively recruited patients with type 2 diabetes and a BMI of $30-35 \mathrm{~kg} / \mathrm{m}^{2}$ [9].

Large-scale observational studies have demonstrated that metabolic surgery reduces the incidence of albuminuria, slows progressive renal functional decline, and reduces the incidence of ESKD in patients with obesity [1013]. Improved control of body weight, blood pressure, dyslipidaemia, and glycaemia contribute to these findings [14]. However, in patients with type 2 diabetes, the antiproteinuric effect of metabolic surgery occurs independently of improvements in body weight, blood pressure, and glycaemia [15]. Therefore, weight-independent renoprotective effects occur after metabolic surgery in people with type 2 diabetes. Synergistic changes in visceral adipose tissue content and location, alterations in adipocytokine signalling, enhanced natriuresis, gut microbiota shifts, and reduced systemic and renal inflammation are purported to play a role $[13,16]$.

Limited access to human kidney tissue is a major limitation of studying human DKD, particularly after metabolic surgery. Pre-clinical studies of metabolic surgery in experimental DKD thus offer a unique opportunity to investigate structural and molecular changes in the kidney postoperatively. In the present review, we aim to summa- rize the renoprotective effects and mechanisms observed in pre-clinical studies of metabolic surgery in rodents with obesity, type 2 diabetes, and kidney disease to date. We supplement the review with additional unpublished findings from our own research group.

\section{Metabolic and Renal Biochemical Parameters}

Table 1 provides an overview of metabolic and renal parameters assessed in pre-clinical studies of metabolic surgery for experimental DKD. Three studies evaluated the impact of RYGB surgery [17-19], 2 evaluated duodenojejunal bypass (DJB) surgery [20,21], and 1 study each utilized Roux-en-Y oesophagojejunostomy (RYEJ) and sleeve gastrectomy (SG) [22, 23]. All experiments studying RYGB were performed in the Zucker diabetic fatty (ZDF) rat model of obesity and DKD by our group. Studies evaluating DJB, RYEJ, and SG were performed in a high-fat diet (40\% calories from fat) plus low-dose streptozotocin (STZ) model of obesity, diabetes, and renal injury in Sprague Dawley or Wistar rats. Xiong et al. [23] evaluated postoperative outcomes at 3 timepoints $(4,8$, and 12 weeks); only data from the final timepoint (12 weeks) are presented in Tables 1 and 2.

\section{Body Weight and Metabolic Control}

RYGB, RYEJ, and SG achieved significant reductions in body weight at timepoints ranging from 7 to 13 weeks postoperatively [17-19, 22, 23]. Although rats undergoing DJB experienced weight gain postoperatively, body weight was reduced compared with sham-operated rats at 8 weeks postoperatively in both studies using this procedure [20,21]. All metabolic surgeries effectively improved glycaemia, with $>70 \%$ reductions in plasma glucose after RYGB reported by Canney et al. [18] and Nair et al. [19]. No studies have assessed glycaemic control after metabolic surgery in experimental DKD using $\mathrm{HbA}_{1 \mathrm{c}}$ or fructosamine. Significant reductions in total cholesterol and triglycerides compared with control rats were reported in a cross-sectional manner at study close after DJB and after RYGB by Zhiqing et al. [20] and Nair et al. [19], respectively. No studies to date have conducted longitudinal pre- and post-metabolic surgery profiling of plasma lipids.

\section{Urinary Protein Excretion and Glomerular Filtration \\ Rate}

RYGB and SG are the only metabolic surgeries that have lowered proteinuria in pre-clinical studies of DKD 

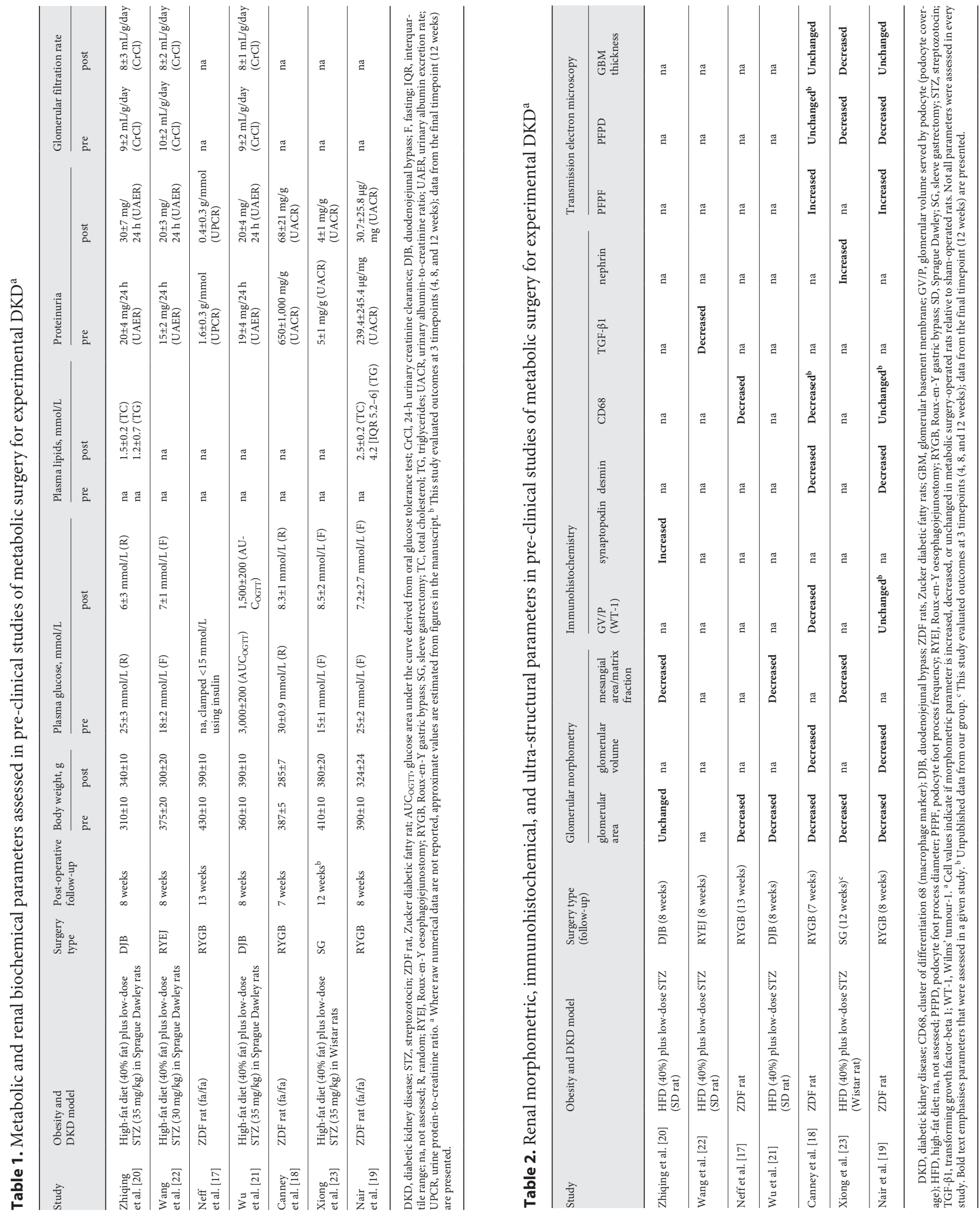
to date $[17-19,23]$ although the magnitude of reduction in proteinuria has been greater with RYGB than SG. Human studies have indicated that RYGB may be more effective than SG in terms of metabolic control [24]. Additionally, more observational and randomized human data exist for RYGB as a renoprotective intervention compared with SG [13]. Certain purported renoprotective effects of metabolic surgery, such as increased natriuresis activating tubuloglomerular feedback to combat glomerular hypertension, exist only for RYGB and not SG $[16,25]$. Stable or increased levels of urinary albumin excretion rate at 8 weeks after DJB were reported by $\mathrm{Wu}$ et al. [21] and Zhiqing et al. [20], respectively. Conversely, Canney et al. [18] reported an $86 \%$ reduction in urinary albumin-to-creatinine ratio compared with sham-operated rats at 7 weeks after RYGB. Similarly, Nair et al. [19] reported an $87 \%$ reduction in preoperative urinary albumin-to-creatinine ratio values at 8 weeks after RYGB. While all studies evaluated urinary protein excretion on timed urinary collections using metabolic cages, there is variability in how results have been reported with researchers using urinary albumin excretion rate, urinary albumin-tocreatinine ratio, and urinary protein-to-creatinine ratio to quantify proteinuria.

Changes in renal function as assessed by measurement of 24-h urinary creatinine clearance have been conducted after RYEJ and after DJB and compared against sham-operated animals. Reductions in creatinine clearance after RYEJ and after DJB relative to sham-operated animals is a consistent finding [20-22]. Limitations of estimating kidney function with serum creatinine after metabolic surgery notwithstanding [26] these findings may reflect remission of glomerular hyperfiltration. Zhiqing et al. [20] also measured serum cystatin $\mathrm{C}$ at 8 weeks after DJB, finding that it was elevated relative to values in sham-operated rats. To date, no studies have directly measured glomerular filtration rate using available methodologies including plasma clearance of iohexol and transcutaneous measurement of FITC-sinistrin clearance $[27,28]$.

\section{Renal Morphometry and Immunohistochemistry}

Table 2 outlines renal morphometric and immunohistochemical parameters assessed in pre-clinical studies of metabolic surgery for DKD to date. Studies have predominantly assessed changes in glomerular structure postoperatively to investigate the structural underpinnings of the pronounced anti-proteinuric effect of metabolic surgery. Reduced glomerular area has been demonstrated by 3 studies evaluating RYGB in ZDF rats [17-19], while Wu et al. [21] and Xiong et al. [23] also demonstrated reductions in glomerular area after DJB and after SG, respectively. Reduced glomerular volume has been exclusively demonstrated after RYGB in 2 studies $[18,19]$, while reduced mesangial matrix expansion has been shown in 2 separate studies of DJB and 1 study of SG but not RYGB $[20,21,23]$. No studies to date have directly assessed renal tubular morphology after metabolic surgery in experimental $\mathrm{DKD}$, which should be a priority for future research in the field given the prominent role assigned to proximal tubular dysfunction in the onset and propagation of proteinuria in DKD.

Immunohistochemistry facilitates the investigation of molecular mechanisms and cell-specific responses within the kidney after metabolic surgery. Staining for Wilms' tumour-1 protein (WT-1), a podocyte-specific nuclear antigen, highlights podocyte distribution and permits calculation of podocyte endowment within the glomerulus [29]. Canney et al. [18] quantified WT-1stained nuclei in the kidney after RYGB. Although no absolute differences in podocyte number were observed, the smaller glomerular volume in RYGB-operated rats resulted in a decrease in the glomerular volume served per podocyte (reduced podocyte coverage). Similarly, Zhiqing et al. [20] demonstrated increased renal expression of another podocyte-specific marker, synaptopodin, 8 weeks after DJB. Xiong et al. [23] demonstrated increased renal expression of nephrin, a key structural protein located at the slit diaphragm area of podocytes, by both immunohistochemistry and Western blotting up to 12 weeks after SG. De novo staining for desmin, a podocyte intermediate filament protein, is an early marker of podocyte mechanical stretch due to glomerular hypertension in the setting of DKD [29]. Canney et al. [18] and Nair et al. [19] both demonstrated significant reductions in the number of desmin-positive cells after RYGB in ZDF rats. Together, these findings suggest that metabolic surgery opposes podocyte dedifferentiation in the setting of DKD.

Neff et al. [17] demonstrated reduced renal expression of the macrophage marker CD68 after RYGB in the ZDF model of DKD. This occurred in parallel with decreased urinary excretion of monocyte chemotactic protein- 1 and improvements in glomerular morphometry and proteinuria. This finding indicates reduced renal inflammation postoperatively, consistent with the observation of reduced urinary excretion of inflammatory cytokines at 
1-year after metabolic surgery in humans [30]. Notably, the RYGB-induced improvements observed by Neff et al. [17] in ZDF rats were recapitulated (save for impact on proteinuria) in a parallel sham-operated group that underwent dietary restriction to achieve RYGB-matched weight loss. Wang et al. [22] demonstrated reduced renal expression of TGF- $\beta 1$ in glomerular and renal tubular epithelial cells at 8 weeks after RYEJ, indicative of an antifibrotic effect of the intervention.

\section{Glomerular Ultrastructure}

While most studies of metabolic surgery for experimental DKD to date have evaluated changes in glomerular morphometry postoperatively, 3 studies ( 2 after RYGB and 1 after SG) have assessed glomerular ultrastructure using transmission electron microscopy (Table 2) $[18,19$, 23]. Injury-associated cytoskeletal rearrangements in podocytes result in the disruption and retraction of primary and secondary foot processes, a phenomenon referred to as foot process effacement. This pattern of glomerular ultra-structural disruption is mechanistically linked to the emergence of proteinuria in DKD through its impact on size-selective sieving properties of the glomerular filtration barrier [31].

Canney et al. [18] demonstrated that RYGB restores normal podocyte foot process frequency (a marker of podocyte health) at 7 weeks after RYGB. Similarly, Nair et al. [19] showed that RYGB restores normal podocyte foot process morphology by increasing foot process frequency and decreasing foot process diameter at 8 weeks after RYGB. Both studies highlighted that RYGB had no effect on glomerular basement membrane (GBM) thickness. As GBM thickness was not significantly elevated in sham-operated versus healthy control rats, power to detect reduced GBM thickening after RYGB was significantly diminished. This latter finding, therefore, reflects a limitation of studying DKD in rodents as not all features of human diabetic nephropathy are reliably recapitulated in rats [32].

Conversely, GBM thickening to approximately 230$250 \mathrm{~nm}$ did develop in Xiong et al.'s [23] study of SG conducted in high-fat diet Wistar rats treated with low-dose STZ. Accordingly, GBM thickness was reduced by SG at 4, 8, and 12 weeks [23]. Similar to Nair et al.'s [19] study of RYGB highlighted above, increased podocyte foot process width (analogous to diameter) which developed in sham-operated rats was reversed by SG out to 12 -week follow-up [23].

Metabolic Surgery for Diabetic Kidney Disease

\section{Renal Cortical Transcriptome}

Nair et al. [19] interrogated changes in the renal cortical transcriptome (assessed using bulk RNA sequencing) at 8 weeks after RYGB in the ZDF rat model. Downstream analysis focused on differentially expressed transcripts with an absolute fold change $\geq 1.3$ and $p$ value adjusted for multiplicity testing (Benjamini-Hochberg) $<0.05$. In total, 379 were genes differentially expressed between sham-operated ZDF rats compared with healthy fa/+ controls, while 942 genes were differentially expressed between RYGB-operated and sham-operated ZDF rats. This corresponded to a change in $2.1 \%(379 / 18,423)$ of the renal transcriptome in sham-operated ZDF rats and $5.1 \%(942 / 18,423)$ of the renal transcriptome in RYGBoperated rats, respectively. Inflammation, tubulopathy, and fibrosis-associated transcripts including Il24 (interleukin-24), Havcr1 (kidney-injury molecule-1), and Spp1 (osteopontin) were strongly increased from health to disease (sham-operated ZDF rats vs. fa/+ rats) and markedly decreased with metabolic surgery (RYGB-operated vs. sham-operated ZDF rats). Additionally, RYGB increased expression of several genes reflecting adaptive responses to postoperative micronutrient deficiency, including Epo and Cyp27b1, indicative of impaired iron and vitamin D homeostasis, respectively.

Pathway enrichment analyses performed using the Reactome database identified upregulation of renal inflammation and fibrosis pathways in sham-operated ZDF rats which was reversed by RYGB $[19,33]$. Conversely, biological oxidation activity was decreased in sham-operated rats and restored by RYGB, reflecting restoration of renal tubular biotransformation capacity postoperatively. Using MCP-counter to estimate renal tissue-infiltrating immune and stromal cell populations [34], RYGB-operated animals were predicted to have decreased immune cell and fibroblast abundance compared with sham-operated ZDF rats.

Of the 379 transcripts differentially expressed from health to disease (sham-operated ZDF rats vs. fa/+ rats), 144 (38.0\%) of these genes were also changed by RYGB. The majority of these genes were changed in the opposite direction to disease-associated transcriptional shifts, emphasizing the corrective impact of RYGB in experimental DKD. Of the 144 disease-associated transcripts corrected by RYGB, 22 were significantly differentially expressed in the glomeruli of patients with DKD [35], indicating the potential of RYGB to decrease DKD-associated inflammation (Csf1r and C4b), TGF- $\beta 1$-mediated fibrosis ( Vim, $F n 1$, and $S p p 1$ ), and adaptive cytoskeletal responses to 
mechanical stretch induced by glomerular hypertension (Tnnt and Tubb6), while also restoring tubular bonemorphogenetic protein-7 signalling (Id4). Indeed, disease-associated transcripts corrected by RYGB strongly and positively correlated with abnormal glomerular morphometry and negatively correlated with podocyte foot process frequency, a marker of glomerular health, suggesting that RYGB-induced corrections in renal inflammation and fibrosis signalling contribute to improved glomerular structure and ultrastructure postoperatively. In particular, TGF- $\beta 1$ signalling pathway-regulated genes including osteopontin (Spp1), vimentin (Vim), and fibronectin $(F n 1)$ strongly correlated with altered glomerular structure. Reduced expression of these targets after RYGB was confirmed by urinary ELISA (Spp1) and qPCR/Western blotting of the renal cortex ( $\mathrm{Vim} / \mathrm{Fn} 1)$. Thus, reduced TGF- $\beta 1$-mediated renal fibrosis emerged as a dominant transcriptomic response to RYGB which persisted through validation in a human DKD glomerular microarray dataset [35], findings which are consistent with reduced renal TGF- $\beta 1$ expression by immunohistochemistry after RYEJ demonstrated by Wang et al. [22].

Elucidating cell-specific responses within the kidney after RYGB has the potential to uncover new mechanisms governing renoprotection. Therefore, we performed a deconvolution analysis of our post-RYGB bulk renal cortical RNA-sequencing data in a publicly available singlecell RNA-sequencing dataset of the human diabetic kidney [36]. Figure 1 presents a heatmap of cell-specific expression patterns of 106 disease-associated transcripts with human orthologs changed by RYGB in our dataset. Interestingly, despite pronounced improvements in glomerular structure and ultrastructure after metabolic surgery in rats, transcriptomic changes are more common in renal tubular segments (particularly the proximal tubule) as well as leucocyte and fibroblast cell populations. This suggests that corrective gene expression changes induced by metabolic surgery in the kidney at sites distant from the glomerulus may contribute to the improved glomerular structure observed.

\section{Translational Relevance of Rodent Models Employed in Pre-Clinical Studies of Metabolic Surgery for DKD}

Rodent models of obesity and diabetes offer a unique opportunity to explore responses to metabolic surgery within the kidney and indeed at the level of the whole organism although findings presented in this review must be interpreted within the context of limitations of pre- clinical modelling of DKD. Mice are the most widely used species in animal research because they breed quickly, are cheap to house, and are amenable to genetic manipulation [32]. However, mortality rates after metabolic surgery in mice are very high, particularly with the RYGB procedure where mortality rates approach $100 \%$ due to technical difficulty fashioning anastomoses [37]. Additionally, the murine forestomach lacks sufficient muscle to push nutrients through the anastomosis after RYGB, resulting in mortality from gastric obstruction [37]. Rats have therefore been favoured for pre-clinical studies of metabolic surgery; postoperative mortality rates with the RYGB procedure in our group have ranged from 10 to $20 \%$.

Rodent models of obesity and diabetes develop glomerular hyper-filtration, albuminuria, and reliably recapitulate histological features of early human diabetic nephropathy but do not develop features of advanced human disease including nodular glomerulosclerosis, marked tubulointerstitial fibrosis, and kidney failure [32]. Although the role of metabolic surgery in the treatment of advanced human DKD is an emerging research question, most human studies of metabolic surgery conducted to date have focused on reducing the incidence of or improving control of early-stage DKD [13]. The severity of kidney disease reiterated by rat models of obesity and diabetes is thus translationally relevant to ongoing human studies in the field.

The ZDF rat utilized in studies of RYGB by our group develops hyperphagia and insulin resistance as a consequence of monogenic obesity due to a homozygous recessive missense mutation in the $\mathrm{fa}$ gene encoding the leptin receptor [38]. Separate to the leptin receptor mutation, the ZDF rat harbours a genetic defect in pancreatic $\beta$-cell gene transcription which contributes to the emergence of type 2 diabetes in the setting of insulin resistance [39]. Importantly, due to the absence of an intact leptin signalling system, ZDF rats do not reliably manifest hypertension [40], which is a critical determinant of DKD progression in humans. ZDF rats also manifest hydronephrosis and progress rapidly to overt diabetes in the adolescent state prior to reaching maturity $[40,41]$. Although ZDF rats manifest obesity, insulin resistance, and dyslipidaemia, they do not develop all features of the human metabolic syndrome and lack a significant period of pre-diabetes which is characteristic of human DKD.

High-fat diet and STZ-treated rats, which have been used by other groups in pre-clinical studies of metabolic surgery, also rapidly progress to overt diabetes without a prolonged period of pre-diabetes as a consequence of STZ 


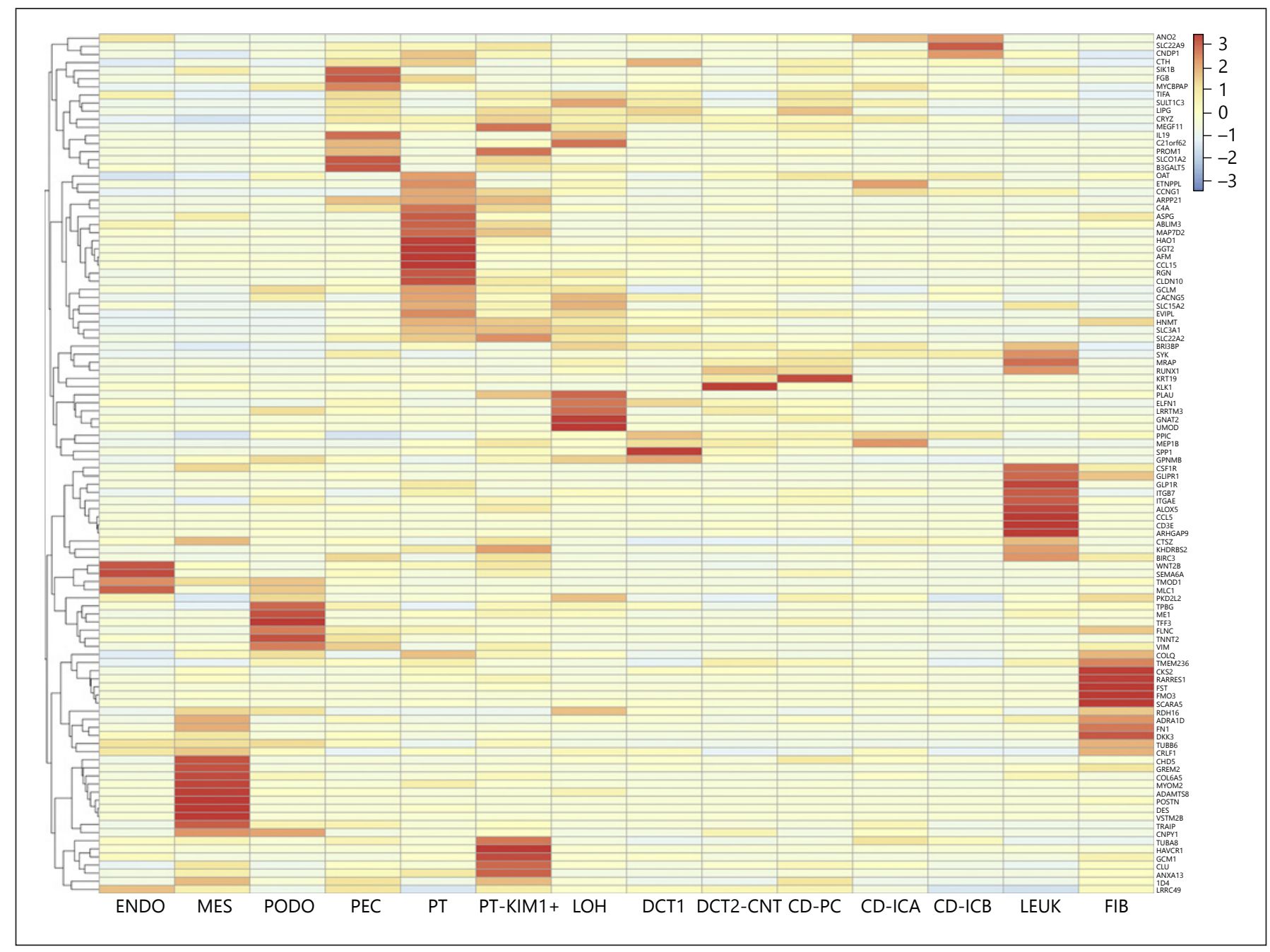

Fig. 1. Deconvolution analysis of $106 \mathrm{DKD}$-associated transcripts which are corrected by RYGB in ZDF rats using publicly available human diabetic kidney single-cell RNA-sequencing data. Columns represent 14 kidney cell types. Cell types include ENDO, MES, PODO, PEC, PT, PT-KIM1+, LOH, DCT1, DCT2-CNT, CD-PC, CD-ICA, CD-ICB, LEUK, and FIB. Rows indicate genes (official gene symbols of human orthologous genes are displayed). Cell colours indicate relative transcript expression levels in the human diabetic kidney: red - high expression in cell type; yellow low expression in cell type; blue - very low/absent expression in

toxicity to pancreatic $\beta$ cells [42]. Similar to the ZDF model, models of STZ-induced diabetes do not reliably manifest hypertension [42]. A distinct disadvantage of STZ is its non-specific renal cytotoxicity which can directly induce renal tubular injury [43]. Although all studies included in the current review utilized a single lowdose of STZ, STZ-induced renal tubular injury has been described even at low doses [44]. High-fat diet and low- cell type. DKD, diabetic kidney disease; RYGB, Roux-en-Y gastric bypass; ZDF rats, Zucker diabetic fatty rats; ENDO, endothelial cells; MES, mesangial cells; PODO, podocytes; PEC, parietal epithelial cells; PT, proximal tubular cells; PT-KIM1+, proximal tubular cell cluster positive for kidney-injury molecule-1; LOH, loop of Henle; DCT1, distal convoluted tubule cluster 1; DCT2-CNT, distal convoluted tubule cluster 2-connecting tubule; $\mathrm{CD}-\mathrm{PC}$, collecting duct-principal cell; CD-ICA, collecting duct-intercalated cell type A; CD-ICB, collecting duct-intercalated cell type B; LEUK, leukocytes; FIB, fibroblasts. dose STZ models may more readily develop manifestations of obesity-related glomerulopathy rather than diabetic nephropathy. However, given the overlap in pathophysiology and renal manifestations of obesity-related glomerulopathy and diabetic nephropathy [45], as well as the growing evidence for metabolic surgery for non-diabetic CKD [13], the findings observed remain translationally relevant. 
Several emerging pre-clinical models of obesity and diabetes overcome many of the aforementioned limitations of the rat models utilized in studies of metabolic surgery to date. The Zucker diabetic Sprague Dawley (ZDSD) rat was developed by crossing lean homozygous ZDF rats with a sub-strain of Sprague Dawley rats that were selectively bred for high-fat diet-induced obesity [46]. Thus, the ZDSD rat combines the defect in pancreatic $\beta$-cell gene transcription characteristic of the ZDF rat with polygenic obesity of the Sprague Dawley rat to produce a model of obesity and diabetes with an intact leptin pathway [46]. The ZDSD rat is thus a more translationally relevant pre-clinical model which spontaneously develops type 2 diabetes and hypertension in the context of polygenic obesity and a prolonged pre-diabetic period. Renal manifestations of the model have also been characterized and include glomerular hyperfiltration, glomerular and tubular injury, mesangial expansion, GBM thickening, and podocyte foot process effacement [47]. The renal physiology and structure of large animals more closely resembles that of the human kidney than smaller animals such as rats. The Iberian pig fed with high-fat diet is a promising model of obesity-related glomerulopathy and diabetic nephropathy, which develops renal histological manifestations that very closely resemble the human disease even in the absence of overt type 2 diabetes, including lipid deposits and some features of advanced glomerular disease such as nodular glomerulosclerosis [48]. These emerging models, which are more translationally relevant to human $\mathrm{DKD}$, come at the expense of prolonged experimental timelines and increased animal husbandry costs.

\section{Conclusions}

All pre-clinical studies of metabolic surgery for experimental DKD have demonstrated pronounced improvements in glycaemia postoperatively. RYEJ, RYGB, and SG achieved postoperative weight loss [17-19,22], while DJB slowed weight gain compared with shamoperated controls [20,21]. RYGB exerted a potent proteinuria-lowering effect across 3 studies [17-19], while DJB and RYEJ slowed progression of proteinuria compared with sham-operated controls [20-22]. SG also lowered proteinuria in a single study although reductions were lesser in magnitude compared with RYGB [23]. No studies to date have examined pre- and postmetabolic surgery changes in dyslipidaemia, blood pressure, and measured glomerular filtration rate, and these should be prioritized by future studies in the field.

Most pre-clinical studies have demonstrated improved glomerular morphometry after metabolic surgery. Immunohistochemical interrogation of the kidney after metabolic surgery has demonstrated improved podocyte endowment (WT-1 and synaptopodin) and structural integrity of podocyte slit diaphragms (nephrin), decreased podocyte mechanical stretch (desmin), and reduced macrophage infiltration and fibrosis (CD68 and TGF- $\beta 1$, respectively) $[17-20,22,23]$. Two studies have used transmission electron microscopy to show that RYGB improves glomerular ultrastructure in ZDF rats $[18,19]$, while similar reductions in podocyte foot process effacement were observed in a single study of SG [23]. No studies have specifically examined changes in renal proximal tubular morphometry and ultrastructure after metabolic surgery in experimental DKD. Such studies should be prioritized as RYGB-induced transcriptional changes are abundant in the proximal tubule, and structural characterization of this tubular segment may uncover new phenomena underpinning the anti-proteinuric effect of metabolic surgery.

Bulk RNA sequencing of the renal cortex has highlighted that RYGB corrects DKD-associated alterations in multiple pathways including fibrosis, inflammation, and biological oxidations. Pro-inflammatory and pro-fibrotic transcripts corrected by RYGB strongly correlate with glomerular structural integrity, providing mechanistic insight into the improved glomerular structure after metabolic surgery. RYGB corrects DKD-associated transcriptomic alterations across all cell types in the kidney, with a predominant effect in glomerular cells, proximal tubular cells, leucocytes, and fibroblasts. Interrogating renal responses to metabolic surgery in experimental DKD using single-cell RNA sequencing should add granularity to mechanisms underpinning its renoprotective effects.

Ultimately, studying renal responses to metabolic surgery in experimental DKD employs a reverse-translational approach whereby mechanisms underpinning the renoprotective effects of metabolic surgery observed in large-scale observational and emerging randomized human studies can be interrogated. Evidence accumulated in pre-clinical studies of metabolic surgery for experimental DKD to date supports a growing role for metabolic surgery in the DKD treatment algorithm. 


\section{Acknowledgements}

We acknowledge local support received in the realization of studies described herein from the University College Dublin (UCD) Biomedical Facility and the Research Pathology and Genomics Core Facilities at the UCD Conway Institute. We thank Dr. Parker Wilson and Dr. Benjamin Humphreys for sharing their human kidney single-cell RNA-sequencing data with us.

\section{Conflict of Interest Statement}

C.W.1.R. discloses personal fees outside of the submitted work from Novo Nordisk, GI Dynamics, Eli Lilly, Johnson and Johnson, Sanofi, Aventis, Astra Zeneca, Janssen, Bristol-Myers Squibb, and Boehringer Ingelheim. The other authors have no conflicts of interest to declare.

\section{Funding Sources}

This work was performed within the Irish Clinical Academic Training (ICAT) Programme, supported by the Wellcome Trust and the Health Research Board (Grant No. 203930/B/16/Z), the Health Service Executive National Doctors Training and Planning, and the Health and Social Care, Research and Development Division, Northern Ireland. The relevant experimental studies conducted in our laboratories have been supported by generous funding from the Science Foundation Ireland (12/YI/B2480) to C.W.1.R., Swedish Medical Research Council (2015-02733) and European Foundation for the Study of Diabetes/Boehringer Ingelheim European Diabetes Research Programme (BI 2017_3) to C.W.1.R. and N.G.D.

\section{Author Contributions}

W.P.M. wrote the manuscript with critical input from C.W.1.R. and N.G.D. All authors reviewed and approved the final manuscript.

\section{References}

1 Saran R, Robinson B, Abbott KC, Agodoa LYC, Bragg-Gresham J, Balkrishnan R, et al. US Renal Data System 2018 Annual Data Report: epidemiology of kidney disease in the United States. Am J Kidney Dis. 2019;73(3 Suppl 1):A7-8.

2 Mann JFE, Ørsted DD, Brown-Frandsen K, Marso SP, Poulter NR, Rasmussen S, et al. Liraglutide and renal outcomes in type 2 diabetes. N Engl J Med. 2017 Aug 31;377(9):839-48.

3 Martin WP, Griffin TP, Lappin DW, Griffin DG, Ferguson JP, O’Brien T, et al. Influence of referral to a combined diabetology and nephrology clinic on renal functional trends and metabolic parameters in adults with diabetic kidney disease. Mayo Clinic Proc Innov Qual Outcomes. 2017 Sep;1(2):150-60.

4 Perkovic V, Jardine MJ, Neal B, Bompoint S, Heerspink HJL, Charytan DM, et al. Canagliflozin and renal outcomes in type 2 diabetes and nephropathy. N Engl J Med. 2019 Jun 13; 380(24):2295-306.

5 Afkarian M, Sachs MC, Kestenbaum B, Hirsch IB, Tuttle KR, Himmelfarb J, et al. Kidney disease and increased mortality risk in type 2 diabetes. J Am Soc Nephrol. 2013; 24(2):302-8.

6 Lim WH, Johnson DW, Hawley C, Lok C, Polkinghorne KR, Roberts MA, et al. Type 2 diabetes in patients with end-stage kidney disease: influence on cardiovascular diseaserelated mortality risk. Med J Aust. 2018 Nov 19;209(10):440-6.

7 Chang AR, Grams ME, Navaneethan SD. Bariatric surgery and kidney-related outcomes. Kidney Int Rep. 2017 Mar;2(2):261-70.

8 Cohen RV, Pereira TV, Aboud CM, Caravatto PP, Petry TB, Correa JL, et al. Microvascular outcomes after metabolic surgery (MOMS) in patients with type 2 diabetes mellitus and class I obesity: rationale and design for a ran- domised controlled trial. BMJ Open. 2017; 7(1):e013574.

9 Cohen RV, Pereira TV, Aboud CM, Petry TBZ, Lopes Correa JL, Schiavon CA, et al. Effect of gastric bypass vs. best medical treatment on early-stage chronic kidney disease in patients with type 2 diabetes and obesity: a randomized clinical trial. JAMA Surg. 2020: e200420.

10 Carlsson LM, Romeo S, Jacobson P, Burza MA, Maglio C, Sjöholm K, et al. The incidence of albuminuria after bariatric surgery and usual care in Swedish Obese Subjects (SOS): a prospective controlled intervention trial. Int J Obes. 2014 May 6;39(1):169.

11 Friedman AN, Wahed AS, Wang J, Courcoulas AP, Dakin G, Hinojosa MW, et al. Effect of bariatric surgery on CKD risk. J Am Soc Nephrol. 2018;29(4):1289-300.

12 Shulman A, Peltonen M, Sjöström CD, Andersson-Assarsson JC, Taube M, Sjöholm K, et al. Incidence of end-stage renal disease following bariatric surgery in the Swedish Obese Subjects study. Int J Obes. 2018 Jun;42(5):964-73.

13 Martin WP, White J, Lopez-Hernandez FJ, Docherty NG, le Roux CW. Metabolic surgery to treat obesity in diabetic kidney disease, chronic kidney disease, and end-stage kidney disease; what are the unanswered questions? Front Endocrinol. 2020;11:289.

14 Holland JA, Martin WP, Docherty NG, Roux CW. Impact of intentional weight loss on diabetic kidney disease. Diabetes Obes Metab. 2019 Oct;21(10):2338-41.

15 Martin WP, le Roux CW. Comment on: metabolic surgery improves renal injury independent of weight loss: a meta-analysis. Surg Obes Relat Dis. 2019 Jun;15(6):1020-3.

16 Martin WP, Docherty NG, Le Roux CW. Impact of bariatric surgery on cardiovascular and renal complications of diabetes: a focus on clin- ical outcomes and putative mechanisms. Expert Rev Endocrinol Metab. 2018 Sep 19;1-12.

17 Neff KJ, Elliott JA, Corteville C, Abegg K, Boza C, Lutz TA, et al. Effect of Roux-en-Y gastric bypass and diet-induced weight loss on diabetic kidney disease in the Zucker diabetic fatty rat. Surg Obes Relat Dis. 2017 Jan; 13(1):21-7.

18 Canney AL, Cohen RV, Elliott JA, CMA, Martin WP, Docherty NG, et al. Improvements in diabetic albuminuria and podocyte differentiation following Roux-en-Y gastric bypass surgery. Diab Vasc Dis Res. 2019 Nov $14 ; 1479164119879039$.

19 Nair M, Martin WP, Zhernovkov V, Elliott JA, Fearon N, Eckhardt H, et al. Characterization of the renal cortical transcriptome following Roux-en-Y gastric bypass surgery in experimental diabetic kidney disease. BMJ Open Diab Res Care. 2020;8(1):e001113.

20 Zhiqing W, Jing W, Haili X, Shaozhuang L, Chunxiao $\mathrm{H}$, Haifeng $\mathrm{H}$, et al. Renal function is ameliorated in a diabetic nephropathy rat model through a duodenal-jejunal bypass. Diabetes Res Clin Pract. 2014 Jan;103(1):26-34.

21 Wu D, Cheng Y-G, Huang X, Zhong M-W, Liu S-Z, Hu S-Y. Downregulation of IncRNA MALAT1 contributes to renal functional improvement after duodenal-jejunal bypass in a diabetic rat model. J Physiol Biochem. 2018 Aug;74(3):431-9.

22 Wang C, He B, Piao D, Han P. Roux-en-Y esophagojejunostomy ameliorates renal function through reduction of renal inflammatory and fibrotic markers in diabetic nephropathy. Obes Surg. 2016 Jul;26(7):1402-13.

23 Xiong Y, Zhu W, Xu Q, Ruze R, Yan Z, Li J, et al. Sleeve gastrectomy attenuates diabetic nephropathy by upregulating nephrin expressions in diabetic obese rats. Obes Surg. 2020 Aug;30(8):2893-904. 
24 Schauer PR, Bhatt DL, Kirwan JP, Wolski K, Aminian A, Brethauer SA, et al. Bariatric surgery versus intensive medical therapy for diabetes: 5-year outcomes. N Engl J Med. 2017; 376(7):641-51.

25 Docherty NG, Fändriks L, le Roux CW, Hallersund P, Werling M. Urinary sodium excretion after gastric bypass surgery. Surg Obes Relat Dis. 2017 Sep;13(9):1506-14.

26 Lopez-Martinez M, Luis-Lima S, Morales E, Navarro-Diaz M, Negrin-Mena N, Folgueras $\mathrm{T}$, et al. The estimation of GFR and the adjustment for BSA in overweight and obesity: a dreadful combination of two errors. Int $J$ Obes. 2020;44(5):1129-40.

27 Schock-Kusch D, Xie Q, Shulhevich Y, Hesser J, Stsepankou D, Sadick M, et al. Transcutaneous assessment of renal function in conscious rats with a device for measuring FITC-sinistrin disappearance curves. Kidney Int. 2011 Jun;79(11):1254-8.

28 Carrara F, Azzollini N, Nattino G, Corna D, Villa S, Cerullo D, et al. Simplified method to measure glomerular filtration rate by iohexol plasma clearance in conscious rats. Nephron. 2016;133(1):62-70.

29 Funk J, Ott V, Herrmann A, Rapp W, Raab S, Riboulet W, et al. Semiautomated quantitative image analysis of glomerular immunohistochemistry markers desmin, vimentin, podocin, synaptopodin and WT-1 in acute and chronic rat kidney disease models. Histochem Cell Biol. 2016 Mar;145(3):315-26.

30 Fenske WK, Dubb S, Bueter M, Seyfried F, Patel K, Tam FW, et al. Effect of bariatric surgery-induced weight loss on renal and systemic inflammation and blood pressure: a 12-month prospective study. Surg Obes Relat Dis. 2013 Jul-Aug;9(4):559-68.

31 Lin JS, Susztak K. Podocytes: the weakest link in diabetic kidney disease? Curr Diab Rep. 2016;16(5):45-.

32 Betz B, Conway BR. An update on the use of animal models in diabetic nephropathy research. Curr Diab Rep. 2016;16(2):18.
$33 \mathrm{Yu} \mathrm{G}, \mathrm{He}$ QY. ReactomePA: an R/bioconductor package for reactome pathway analysis and visualization. Mol Biosyst. 2016 Feb; 12(2):477-9.

34 Becht E, Giraldo NA, Lacroix L, Buttard B, Elarouci N, Petitprez F, et al. Estimating the population abundance of tissue-infiltrating immune and stromal cell populations using gene expression. Genome Biol. 2016 Oct 20; 17(1):218

35 Woroniecka KI, Park AS, Mohtat D, Thomas DB, Pullman JM, Susztak K. Transcriptome analysis of human diabetic kidney disease. Diabetes. 2011 Sep;60(9):2354-69.

36 Wilson PC, Wu H, Kirita Y, Uchimura K, Ledru N, Rennke HG, et al. The single-cell transcriptomic landscape of early human diabetic nephropathy. Proc Natl Acad Sci U S A. 2019 Sep 24;116(39):19619-25.

37 Yin DP, Gao Q, Ma LL, Yan W, Williams PE, McGuinness OP, et al. Assessment of different bariatric surgeries in the treatment of obesity and insulin resistance in mice. Ann Surg. 2011;254(1):73-82.

38 Phillips MS, Liu Q, Hammond HA, Dugan V, Hey PJ, Caskey CJ, et al. Leptin receptor missense mutation in the fatty Zucker rat. Nat Genet. 1996 May;13(1):18-9.

39 Griffen SC, Wang J, German MS. A genetic defect in beta-cell gene expression segregates independently from the fa locus in the ZDF rat. Diabetes. 2001 Jan;50(1):63-8.

40 Vora JP, Zimsen SM, Houghton DC, Anderson S. Evolution of metabolic and renal changes in the ZDF/Drt-fa rat model of type II diabetes. J Am Soc Nephrol. 1996 Jan;7(1): 113-7.

41 Szöcs Z, Brunmair B, Stadlbauer K, Nowotny $\mathrm{P}$, Bauer L, Luger A, et al. Age-dependent development of metabolic derangement and effects of intervention with pioglitazone in Zucker diabetic fatty rats. J Pharmacol Exp Ther. 2008 Jul;326(1):323-9.
42 Kitada M, Ogura Y, Koya D. Rodent models of diabetic nephropathy: their utility and limitations. Int J Nephrol Renovasc Dis. 2016;9: 279-90.

43 Tay YC, Wang Y, Kairaitis L, Rangan GK, Zhang C, Harris DC. Can murine diabetic nephropathy be separated from superimposed acute renal failure? Kidney Int. 2005;68(1): 391-8.

44 Kraynak AR, Storer RD, Jensen RD, Kloss MW, Soper KA, Clair JH, et al. Extent and persistence of streptozotocin-induced DNA damage and cell proliferation in rat kidney as determined by in vivo alkaline elution and BrdUrd labeling assays. Toxicol Appl Pharmacol. 1995 Dec 1;135(2):279-86.

45 D'Agati VD, Chagnac A, de Vries APJ, Levi $M$, Porrini E, Herman-Edelstein $M$, et al. Obesity-related glomerulopathy: clinical and pathologic characteristics and pathogenesis. Nat Rev Nephrol. 2016 Aug 1;12(8):453-71.

46 Peterson RG, Jackson CV, Zimmerman K, de Winter W, Huebert N, Hansen MK. Characterization of the ZDSD rat: a translational model for the study of metabolic syndrome and type 2 diabetes. J Diabetes Res. 2015;2015: 487816.

47 Peterson RG, Jackson CV, Zimmerman KM. The ZDSD rat: a novel model of diabetic nephropathy. Am J Transl Res. 2017;9(9):4236-49.

48 Rodríguez RR, González-Bulnes A, GarciaContreras C, Elena Rodriguez-Rodriguez A, Astiz S, Vazquez-Gomez M, et al. The Iberian pig fed with high-fat diet: a model of renal disease in obesity and metabolic syndrome. Int $\mathrm{J}$ Obes. 2020 Feb;44(2):457-65.

49 Martin WP, Bauer J, Coleman J, DellatorreTeixeira L, Reeve JLV, Twomey PJ, et al. Obesity is common in chronic kidney disease and associates with greater antihypertensive usage and proteinuria: evidence from a cross-sectional study in a tertiary nephrology centre. Clin Obes. 2020 Dec;10(6):e12402. 